\title{
ON THE STRUCTURE OF MAXIMALLY ALMOST PERIODIC GROUPS
}

\author{
BY THEODORE W. WILCOX
}

\author{
Communicated by Richard Arens, May 16, 1967
}

1. Introduction. A topological group $G$ is said to be maximally almost periodic if the continuous almost periodic functions separate points in $G$, or equivalently if the continuous finite-dimensional unitary representations of $G$ separate points in $G$. See [4], or [2, §18]. Throughout this note, we use "representation" to mean "continuous finite-dimensional unitary representation". Our purpose here is to announce some results concerning maximally almost periodic (MAP) groups which are independent of the classical theorem of FreudenthalWeil which states that a locally compact connected group is MAP if and only if it is the direct product of $\boldsymbol{R}^{n}$ and a compact group $[6, \S \S 30,31]$.

The results in this note comprise a portion of the author's doctoral dissertation. Detailed proofs of these and other results will appear at a later date. The author thanks his thesis advisor, Professor Edwin Hewitt, and Professor Lewis Robertson for all their assistance and encouragement. ${ }^{1}$

2. Definitions and notation. Let $K$ be a (Hausdorff but not necessarily locally compact) topological group, $G$ a normal subgroup of $K$ and $T=\{t(x): x \in K\}$ be the group of topological automorphisms of $G$ which are restrictions to $G$ of inner automorphisms of $K$. Let $\hat{K}$ (and $\hat{G}$ resp.) be the space of equivalence classes of irreducible representations of $K$ (and $G$ resp.). In an investigation of $\hat{K}$ it is natural to consider the action on $\hat{G}$ induced by $T$. For example, see [1]. Let $U$ be a representation, $U \in \sigma \in \hat{G}$, define $t^{*}(x) U=U \circ t(x)^{-1}$ and define $t^{*}(x) \sigma$ to be the equivalence class of $t^{*}(x) U$. If the set $\left\{t^{*}(x) \sigma\right.$ : $t(x) \in T\}$ is finite, then $\sigma$ is said to be finitely orbited by $T$. Let $F(\hat{G}, T)$ be the set $\{\sigma \in \hat{G}: \sigma$ is finitely orbited by $T\}$. The von Neumann kernel of a group is the intersection of all kernels of representations of that group.

\section{Results.}

Theorem 1. Let $K, G$ and $T$ be as above. If $U \in \sigma \in \hat{K}$ and if $y \in G$ are such that $U_{y} \neq I$, then there exists an element of $F(\hat{G}, T)$ which separates

1 This research was supported by a NASA Predoctoral Traineeship at the University of Washington. 
$y$ from the identity. In particular, if $K$ is $M A P$, then $F(\hat{G}, T)$ separates points in $G$.

This is proved by utilizing the uniqueness of the decomposition into a direct sum of irreducible constituents of the restriction of $U$ to $G$; the equivalence classes of these constituents are permuted by the action of $T$.

Theorem 2. Let $K, G$ and $T$ be as above. Let $\sigma \in F(\hat{G}, T)$ and let $O(\sigma, T)$ be the orbit of $\sigma$ by $T$. Then the mapping $\Sigma$ which sends $x$ onto the restriction of $t^{*}(x)$ to $O(\sigma, T)$ is well defined and is a continuous homomorphism of $K$ onto a finite group. The kernel of $\Sigma$ contains $G$.

In general the condition that $F(\hat{G}, T)$ separate points in $G$ is not enough to imply that $K$ is MAP even if $K / G$ is assumed to be MAP. However, if $K$ is the semidirect product of $G$ and a topological group $H, K=G$ (s) $_{\beta} H$, then we have

Theorem 3. Let $K=G \S_{\beta} H$. Let $H_{0}\left(\right.$ and $\left(G \S_{\beta} H\right)_{0}$ resp.) be the von Neumann kernel of $H$ (and $G \bigodot_{\beta} H$ resp.). Let $S=\bigcap\{$ ker $U$ : $U \in \sigma \in F(\hat{G}, \beta(H))\}$. Then $\left(G \Im_{\beta} H\right)_{0}=S \subseteq H_{0}$. In particular, $G \S_{\beta} H$ is $M A P$ if and only if $H$ is $M A P$ and $F(\hat{G}, \beta(H))$ separates points in $G$.

The connection between $\beta(H)$ here and the $T$ above follows from the equation $t(e, h)(x, e)=(\beta(h)(x), e)$. See [2, p. 7]. The major diffculty in the proof of this theorem is to show that if $g \in G$ and if $U \in \sigma \in F(\hat{G}, \beta(H))$ are such that $U_{g} \neq I$, then there exists a representation $V$ of $K$ which separates $(g, e)$ from the identity. A rough sketch follows. Let $\Sigma$ be the homomorphism corresponding to $\sigma$ defined in Theorem 2. Then $\operatorname{ker} \Sigma=G(S)$ and $(G \odot H) /(G(M)$ is a finite group. Let $\mathfrak{U}(n)$ be the unitary group of $U$ and use Burnside's theorem $\left[3\right.$, p. 276] to know that the set $\left\{U_{x}: x \in G\right\}$ spans the $n^{2}$-dimensional Hilbert space of all linear operators on $\boldsymbol{C}^{n}$ ( $\boldsymbol{C}$ is the field of complex numbers). A closed subgroup $\mathfrak{U}$ of $\mathfrak{U}\left(n^{2}\right)$, a semidirect product $\mathfrak{U}(n) \subseteq \mathfrak{U}$ and a continuous homomorphism $\phi$ of $G$ (s) $M$ into $\mathfrak{U}(n)$ (s) are constructed. Then $\phi(g, e)$ can be separated from the identity by a representation $W$ of the compact group $\mathfrak{U}(n) \subseteq \mathfrak{U}$ and the desired representation $V$ of $K$ is induced from the representation $W \circ \phi$ of $\operatorname{ker} \Sigma$.

If $G$ is an Abelian group, then we can identify the character group $X$ of $G$ with $\hat{G}$ and with the notation as in 2 above, $F(X, T)$ is a subgroup of $X$.

TheOrem 4. Let $V$ be a normal subgroup of a topological group $K$. Assume further that $V$ is topologically isomorphic to the additive group 
of a finite-dimensional vector space over some locally compact, nondiscrete field of characteristic zero. Let $C$ be the centralizer of $V$ in $K$. Then $K$ is $M A P$ if and only if $C$ is $M A P$ and $K / C$ is a finite group.

We make use of Pontrjagin's classification of locally compact fields [5, Satz 22] and the fact that the field of real numbers and the $p$-adic number fields are self-dual to show that the finitely orbited characters of $V$ form a subspace of $V$, so that $F(V, T)$ is closed in $V$. Furthermore, it follows from Theorem 1 that $F(V, T)$ is dense in $V$. These facts imply that $T$ must be finite so that $C$ must have finite index in $K$.

Using a $p$-series field, a group can be constructed to show that the hypothesis above (that the field have characteristic zero) is essential.

\section{REFERENCES}

1. R. J. Blattner, Group extension representations and the structure space, Pacific J. Math. 15 (1965), 1101-1113.

2. E. Hewitt and K. A. Ross, Abstract harmonic analysis. I, Springer, Berlin, 1963.

3. N. Jacobson, Lectures in abstract algebra. II, van Nostrand, Princeton, 1953.

4. J. von Neumann, Almost periodic functions in a group. I, Trans. Amer. Math. Soc. 36 (1934), 445-492.

5. L. S. Pontrjagin, Topologische Gruppen, Teil I, Teubner, Leipzig, 1957.

6. A. Weil, L'integration dans les groupes topologiques, deuxième édition, Actualités Sci. Indust. 1145, Hermann, Paris, 1965.

UNIVERSITY OF WASHINGTON 